\title{
Spinal intradural extramedullary cavernous hemangioma with hemorrhagic transformation
}

\author{
Gyu Hyun Kang, Dong Wuk Son, Su Hun Lee, Jun Seok Lee, Sang Weon Lee, Geun Sung Song \\ Department of Neurosurgery, Pusan National University Yangsan Hospital, Pusan National University School of Medicine, Yangsan, Korea
}

\begin{abstract}
Cavernous hemangioma in the spine is rare, and the occurrence of intradural extramedullary cavernous hemangioma is particularly infrequent. A 57-year-old male patient visited the emergency room with suddenly worsening neurological symptoms. Computed tomography at the T10-11 levels showed a round isodense mass lesion, and magnetic resonance imaging showed a 1.2-cm oval intradural extramedullary mass. Therefore, the diagnosis of an intradural extramedullary tumor was suspected. Active subdural hemorrhage was seen when the dura was opened after T10-11 laminectomy. The tumor had brown and reddish colorations. The diagnosis of cavernous hemangioma was made based on a pathological examination, which showed a dilated and congested vascular space lined by endothelial cells. Hemorrhagic transformation of a cavernous hemangioma can worsen a patient's condition. Therefore, before surgery, we should consider the possibility of other tumors, such as cavernous hemangiomas, as well as common intradural extramedullary tumors.
\end{abstract}

Keywords: Cavernous hemangioma; Spinal cord neoplasm

\section{Introduction}

Cavernous hemangioma accounts for $5 \%$ to $12 \%$ of all spinal vascular anomalies [1] and mainly occur in the vertebral body. Therefore, intradural extramedullary cavernous hemangioma are rare compared with vertebral body, epidural, and intramedullary cavernous hemangioma [2], with hemorrhagic transformation being very rare. Hemorrhagic transformation exacerbates the patient's neurological symptoms. In our case, the patient had a sudden neurological deterioration. Here, we report a case of hemorrhagic transformation of cavernous hemangioma presenting with sudden paraplegia, and have added the pertinent literature review.

\section{Case Report}

A 57-year-old male with a week-long low back pain and right radi-

Received: February 9, 2021

Revised: March 12, 2021

Accepted: March 24, 2021

Corresponding Author: Dong Wuk Son, MD

Department of Neurosurgery, Pusan National University Yangsan Hospital, 20 Geumo-ro, Mulgeum-eup, Yangsan 50612, Korea

Tel: +82-55-360-2126; Fax: +82-55-360-2156; E-mail: md6576@naver.com ating pain had been treated at a local hospital. However, the patient was admitted to our emergency room, presenting with paraplegia that suddenly occurred 3 days prior. During physical examination, the motor power of the right lower extremity was at Medical Research Council (MRC) grade 3. Sensory deficit was at hypoesthesia below the T11 level.

Bowel and bladder function were normal, as well as the rectal examination results. The patient's previous medication, medical history, and family history were unremarkable. Computed tomography at the T10-11 level showed a round isodense mass lesion. Magnetic resonance imaging (MRI) showed 1.2- $\mathrm{cm}$ oval intradural extramedullary mass. The T1-weighted image exhibited an isosignal (Fig. 1A), whereas the T2-weighted image showed a heterogenous high signal on the sagittal and axial views (Fig. 1B, 1C). Moreover, the contrast-enhanced T1-weighted image showed homogenous enhancement (Fig. 1D). The MRI signals of cavernous hemangiomas can exhibit various signals depending on the hemorrhage period. Therefore, we suspected a differential diagnosis of schwannomas and meningiomas, with high incidence. Because of the sudden neurological symptom, emergency surgery was performed under neuromonitoring. After performing T10-11 laminectomy and upon the opening of the dura, the tumor exhibited 

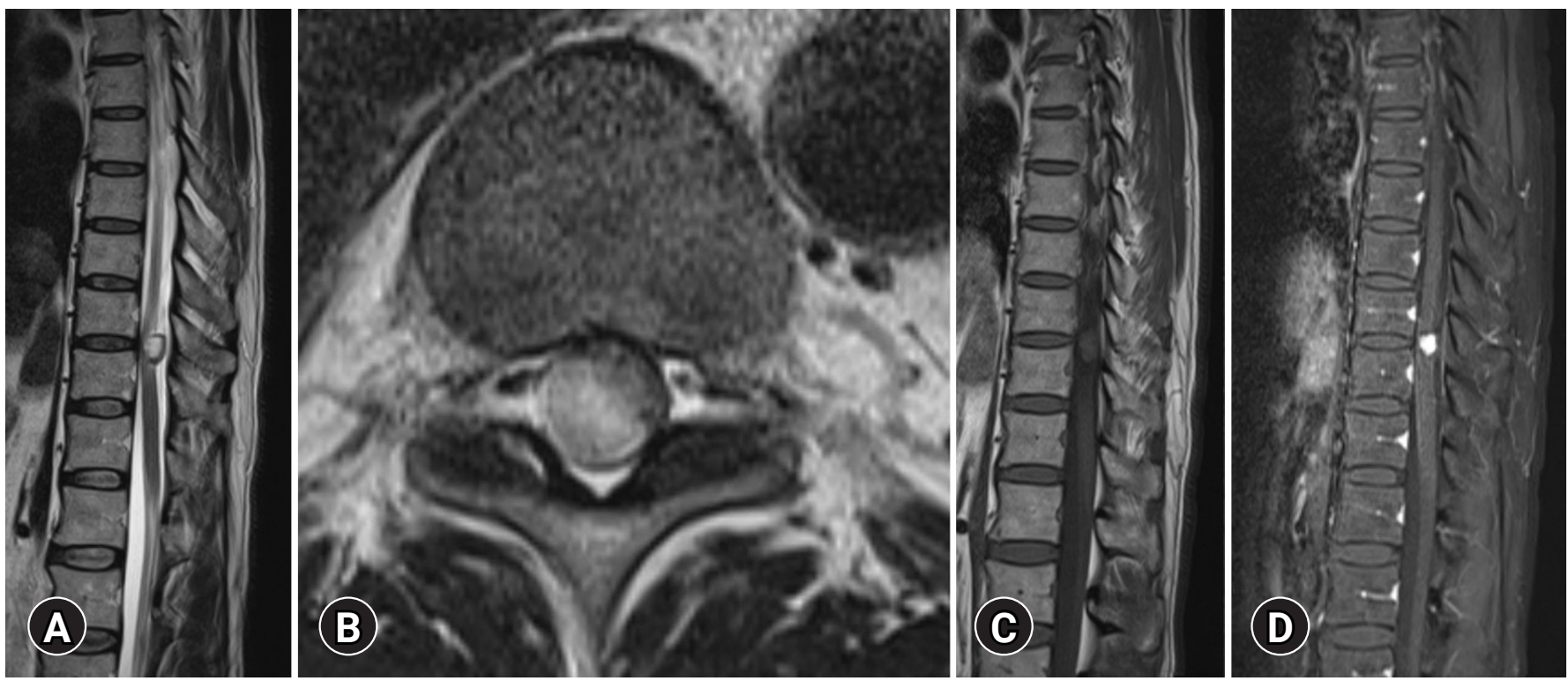

Fig. 1. Magnetic resonance imaging (MRI) shows an intradural extramedullary mass with a 1.2-cm oval shape. (A) A T1-weighted image shows iso-signal intensity. $(B, C)$ T2-weighted images show a heterogeneous high signal. (D) A contrast-enhanced T1-weighted image shows homogeneous enhancement.

active bleeding in the subdural space. The tumor showed brown and reddish overall coloration. An en bloc resection was subsequently performed (Fig. 2). After surgery, the patient improved to an MRC grade 4. The diagnosis of cavernous hemangioma was made during pathological examination, which showed dilated and congested vascular space lined by endothelial cells (Fig. 3).

This study was reviewed and approved by the Institutional Review Board of Pusan National University Yangsan Hospital (IRB No. 05-2021-111). Due to its retrospective nature, the study was exempt from requiring informed consent from the participants.

\section{Discussion}

Spinal intradural extramedullary tumors account for two-thirds of all spinal tumors. Of these, $50 \%$ are meningiomas, $40 \%$ are schwannomas, and $10 \%$ are other tumors $[3,4]$. In the spine, the majority of cavernous hemangiomas are found in the vertebrae, with approximately $3 \%$ being intradural, and most of intradural cavernous hemangiomas being intramedullary [5]. Extramedullary tumors are hence very rare, and hemorrhagic transformation of hemangiomas rarely cause sudden neurological symptoms.

Hemangioma is a histological feature divided into capillary hemangioma and cavernous hemangioma. Cavernous hemangio$\mathrm{ma}$ is a benign vascular lesion with an irregular sinusoidal dilated vascular channel that has a thin collagenous wall. Moreover, it has a single layer of endothelium without an arterial feature, and no elastic and smooth muscle fibers can be observed on the blood vessel

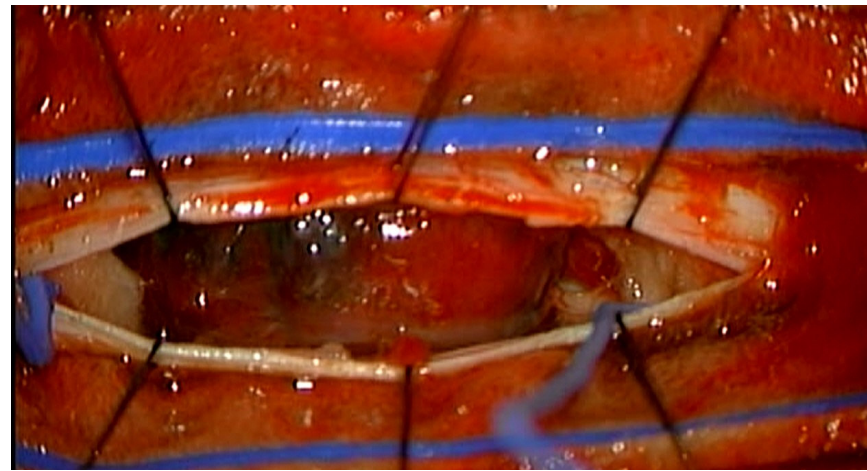

Fig. 2. Intraoperative image of the tumor. After performing laminectomy at T10-11, dura opening showed active subdural hemorrhage. The tumor had an overall brown and reddish coloration.

walls [6-8]. In the present case, histologically cavernous hemangioma was diagnosed with a dilated and congested vascular space lined by endothelial cells.

In the spinal intradural extramedullary space, these tumors may arise from the blood vessels of the nerve roots in the cauda equina, the inner surface of the dura, or the pial surface of the spinal cord [5]. In the present case, the dura attachment site was checked (Fig. 4). Therefore, we thought of it as an intradural extramedullary tumor.

Intradural extramedullary cavernous hemangioma is mostly male dominant and occurs mainly at the thoracic level. Most clinical symptoms are accompanied by back pain and exhibit radiculopathy. It is also characterized by space-occupying masses, but the 


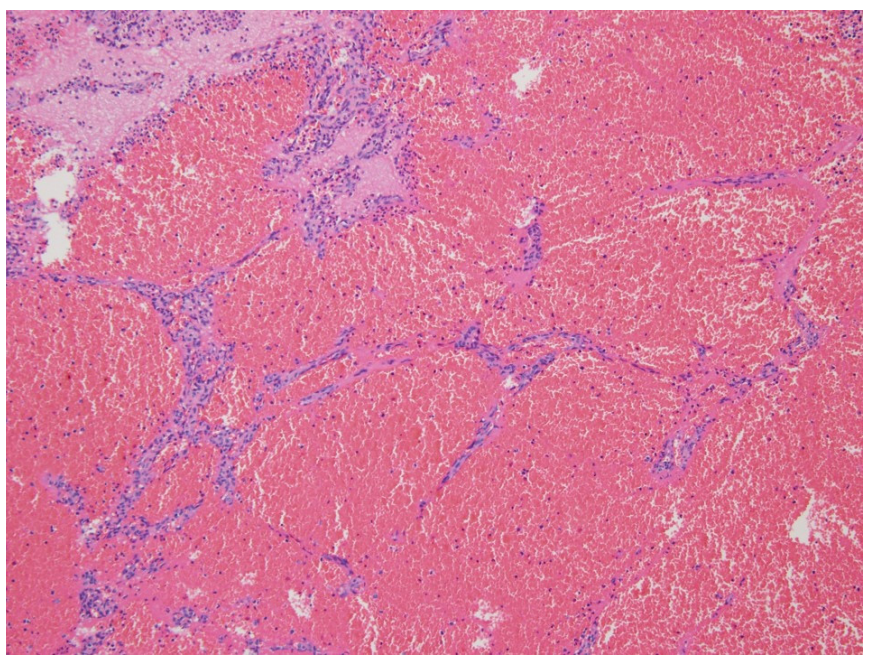

Fig. 3. Pathologic examination. Cavernous hemangioma was diagnosed, with a dilated and congested vascular space lined by endothelial cells.

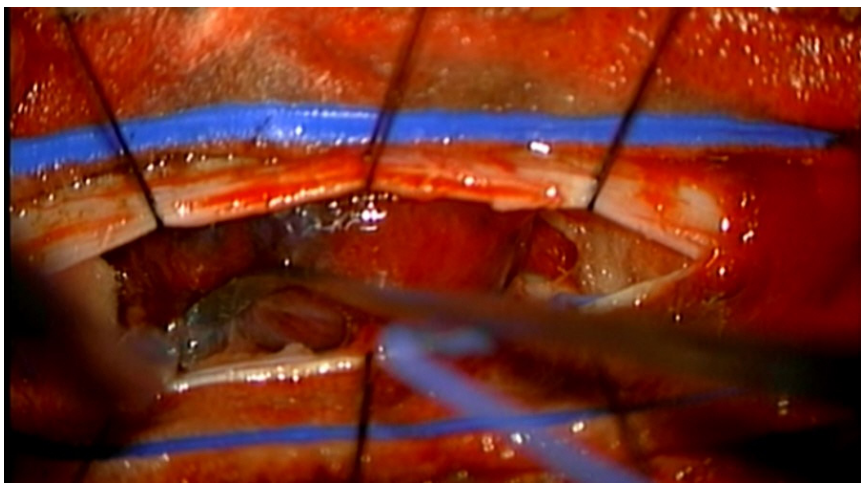

Fig. 4. Intraoperative image. The tumor appears attached to the dura mater.

clinical course is slow. However, due to cord compression, myelopathy and radiculopathy symptoms are accompanied by a progressive motor and sensory deficit. In rare cases, acute hemorrhage may cause sudden paraplegia symptoms [2]. The present case exhibited sudden symptoms of paraplegia due to an uncommon cavernous hemangioma hemorrhage transformation. Nevertheless, the cause of this hemorrhagic transformation is yet to be determined. The most common causes of transformation are coagulopathies, hypertension, increased venous pressure, and vascular malformation [9].

MRI is the gold standard for diagnosis. In the present case, meningioma and schwannoma were differentially diagnosed during preoperative MRI. Cavernous hemangioma generally exhibit various signals on T1- and T2-weighted images depending on blood flow status, calcification, and hemosiderin status. T2-weighted images show heterogeneous and hyperintense signals, and are more sensitive to hemorrhage. By contrast, calcium contrast-enhanced images generally show low signals, but could exhibit variability with factors such as hemorrhage, hemosiderin, and calcification. Spinal intradural extramedullary cavernous hemangioma have been reported in 30 cases, 10 of which presented with spinal subarachnoid hemorrhage [10]. In the present case, subarachnoid hemorrhage was not observed and acute subdural hemorrhage was seen instead. Jin et al. [11] reported that $25 \%$ of surgical patients with spinal intradural extramedullary cavernous hemangioma did not have improved symptoms. In the present case, total excision was implemented. The patient's symptoms improved and he was then discharged.

Only the surgical removal of the tumor can improve symptoms and prevent disease progression. Moreover, when the neurological symptoms worsen, surgery should be hastened.

The present case is an unusual intradural extramedullary cavernous hemangioma hemorrhage transformation, which required accurate diagnosis and rapid surgery due to the sudden exacerbation of the patient's neurological symptoms. However, cavernous hemangioma is difficult to differentiate from other tumors using MRI. If intradural extramedullary tumor is suspected and surgery is implemented, cavernous hemangioma should be considered if some non-specific signal appears in the MRI. Moreover, rapid treatment seems to have a positive impact on the patient's future prognosis.

\section{Conflicts of interest}

No potential conflict of interest relevant to this article was reported.

\section{ORCID}

Gyu Hyun Kang, https://orcid.org/0000-0001-5838-2193

Dong Wuk Son, https://orcid.org/0000-0002-9154-1923

Su Hun Lee, https://orcid.org/0000-0001-8952-5556

Jun Seok Lee, https://orcid.org/0000-0003-2488-6953

Sang Weon Lee, https://orcid.org/0000-0002-3199-7072

Geun Sung Song, https://orcid.org/0000-0001-8029-9011

\section{REFERENCES}

1. Pia HW, Djindjian R. Spinal angiomas: advances in diagnosis and therapy. Berlin Heidelberg: Springer-Verlag Berlin Heidelberg; 1978.

2. Mori K, Ishii H, Tomita Y, Nakajima K, Morimoto K, Maeda M. Intradural-extramedullary spinal cavernous angioma: case re- 
port. Neurol Med Chir (Tokyo) 1991;31:593-6.

3. Albanese V, Platania N. Spinal intradural extramedullary tumors: personal experience. J Neurosurg Sci 2002;46:18-24.

4. Merhemic Z, Stosic-Opincal T, Thurnher MM. Neuroimaging of spinal tumors. Magn Reson Imaging Clin N Am 2016;24: 563-79.

5. Nozaki K, Inomoto T, Takagi Y, Hashimoto N. Spinal intradural extramedullary cavernous angioma: case report. J Neurosurg 2003;99):316-9.

6. Duke BJ, Levy AS, Lillehei KO. Cavernous angiomas of the cauda equina: case report and review of the literature. Surg Neurol 1998;50:442-5.

7. Fontaine S, Melanson D, Cosgrove R, Bertrand G. Cavernous hemangiomas of the spinal cord: MR imaging. Radiology 1988;
166:839-41.

8. Hadlich R. Ein fall von tumor cavernosus des rückenmarks mit besonderer berücksichtigung der neueren theorien über die genese des cavernoms. Virchow Arch Path Anat 1903;172:429-45.

9. Jo BJ, Lee SH, Chung SE, et al. Pure epidural cavernous hemangioma of the cervical spine that presented with an acute sensory deficit caused by hemorrhage. Yonsei Med J 2006;47:877-80.

10. Katoh N, Yoshida T, Uehara T, Ito K, Hongo K, Ikeda S. Spinal intradural extramedullary cavernous angioma presenting with superficial siderosis and hydrocephalus: a case report and review of the literature. Intern Med 2014;53:1863-7.

11. Jin YJ, Chung SB, Kim KJ, Kim HJ. Spinal intradural extramedullary cavernoma presenting with intracranial superficial hemosiderosis. J Korean Neurosurg Soc 2011;49:377-80. 